\title{
Identifying patients at high risk of breast cancer recurrence: strategies to improve patient outcomes
}

This article was published in the following Dove Press journal:

Breast Cancer: Targets and Therapy

8 October 2015

Number of times this article has been viewed

\author{
Yehoda M Martei' \\ Jennifer M Matro',2 \\ 'Department of Medicine, \\ Hematology-Oncology Division, \\ ${ }^{2}$ Abramson Cancer Center, University \\ of Pennsylvania, Philadelphia, PA, USA
}

\begin{abstract}
Identifying patients at high risk of breast cancer recurrence has important implications not only for enabling the ability to provide accurate information to patients but also the potential to improve patient outcomes. Patients at high recurrence risk can be offered appropriate treatment to improve the overall survival. However, the major challenge is identifying patients with early-stage breast cancer at lower risk who may be spared potentially toxic therapy. The successful integration of molecular assays into clinical practice may address the problem of overtreatment and improve overall patient outcomes.
\end{abstract}

Keywords: breast cancer, early stage, genomic assays, recurrence risk, recurrence score

\section{Introduction}

Accurate estimates regarding recurrence and survival are critical for selecting patients with breast cancer who will benefit from adjuvant therapy. This is especially important in patients with early breast cancer, who now make up the majority of patients due to widespread uptake of screening mammography. Decisions about the type of treatment have traditionally been based on the histopathologic parameters, including lymph node status, histologic grade, and tumor size. However, these characteristics fail to characterize the biologic heterogeneity of tumors, which has important implications for treatment benefit. ${ }^{1-7}$

The advent of microarray gene expression profiles as well as sequencing of the whole genome has brought several multigene platforms into clinical use. Many of these platforms incorporate traditional markers (eg, estrogen receptor [ER], progesterone receptor [PR], and human epidermal growth factor receptor 2 [HER2]/neu) as well as additional cancer-associated genes. Genomic assays are increasingly being incorporated into treatment planning for patients with early-stage breast cancer to provide both prognostic and predictive information. These assays supplement the traditional histopathologic markers and help identify patients at high risk of recurrence. They also provide a more quantitative approach to risk assessment and enable individualization of treatment. This has both quality of life and health care cost implications, because patients who will not benefit from a certain treatment can be spared both the toxicity and the expense. ${ }^{8}$

The objective of this review is to provide an overview of the traditional histopathologic features used in prognosticating breast cancer recurrence and to describe some of the newer genomic assays that are now available in the clinical setting to estimate recurrence risk and allow for individualization of treatment and improvement in patient outcomes. This review focuses on the major commercialized molecular assays that 
are most frequently used in clinical practice in the USA and Europe and that have been the most extensively studied.

\section{Histopathologic parameters}

Traditional clinicopathologic features, including lymph node status, tumor size, histologic grade, histologic subtype, patient age, and ER/PR status, have been used in clinical practice for years to estimate prognosis and to predict benefit from various treatment options. A review of 21 pathologic and five clinical features in the National Surgical Adjuvant Breast and Bowel Project B-04 (NSABP B-04) cohort of 620 patients identified tumor size, number of nodal metastases, and nipple involvement as independent prognostic discriminants. ${ }^{9}$ The results of NSABP B-06, which established lumpectomy plus radiation as an appropriate alternative to total mastectomy in stage I and II breast cancer, highlight similar trends, with increase in the number of lymph nodes associated with worsening survival. ${ }^{10,11}$ Additionally, in a review of data from 644 patients treated with mastectomy with median follow-up of 18.2 years, Rosen et al identified tumor size, perimenopausal status, number of axillary lymph node metastases, poorly differentiated grade, presence of lymphatic invasion, and an intense lymphoplasmacytic reaction around the tumor as negative prognostic features. For instance, they estimated that the local risk of recurrence at 20 years for T1N0 and T1N1 tumors was $2.8 \%$ and $6.5 \%$, respectively. ${ }^{12}$ Historic data suggest a 10 -year disease-free survival of $\sim 60 \%-75 \%$ in node-negative cancers compared to $25 \%-30 \%$ in nodepositive cancers.

Other prognostic information is derived from response to systemic chemotherapy in patients who receive preoperative ("neoadjuvant") chemotherapy. In the NSABP B-18 and NSABP B-21 trials, patients who do not achieve a pathologic complete response ( $\mathrm{pCR}$ ) were at higher risk of recurrence compared to patients who achieve pCR. ${ }^{13,14}$ Currently, this information is purely prognostic and the potential benefit of tailoring systemic chemotherapy (ie, switching therapy in patients with limited response) or offering additional chemotherapy if patients do not achieve pCR is unknown.

In patients with less common histologic subtypes, small population studies and retrospective analyses suggest correlation between histology and outcomes, which can also aid with prognostication. These rare histologies include tubular, cribriform, and mucinous carcinoma subtypes associated with favorable outcomes and metaplastic carcinoma and highgrade neuroendocrine cancer of the breast associated with poor outcomes. ${ }^{15-17}$
Hormone receptor and HER2 testing provide additional predictive information that allows for tailoring therapy. Determining the incremental benefit of adjuvant systemic chemotherapy beyond hormone therapy in patients with early-stage breast cancer remains a challenge. Features that predict a high rate of distant recurrence include primary tumor size, close margins or pathologic positive margins, high nuclear grade, age $<35$ years old, and HER2 positive or HR negative. ${ }^{18,19}$ HER2-positive status provides prognostic information in node-negative breast cancer as well as predicting information in selecting targeted and systemic therapy. ${ }^{20}$ Moreover, in patients with node-positive disease who are at increased risk of recurrence, the predictive value for adjuvant chemotherapy appears to be more established. ${ }^{21}$

\section{Adjuvant!}

Adjuvant! (Adjuvant! Inc., San Antonio, TX, USA) is a standardized decision tool that uses classical clinicopathologic features to estimate 10-year recurrence risk and mortality among patients with early-stage breast cancer with and without adjuvant therapy. ${ }^{22}$ Risk estimates were obtained from the Surveillance, Epidemiology, and End Results registry and are based on 10-year overall survival for women age 36-69 years diagnosed with breast cancer between 1988 and 1992. Breast cancer-specific survival among untreated patients was adjusted for the estimated frequency of the use of systemic therapy and expected efficacy of that therapy. Event-free survival estimates were derived from Surveillance, Epidemiology, and End Results mortality data, and the efficacy of various chemotherapy and endocrine therapies was derived from the Early Breast Cancer Trialists' Collaborative Group 1998 meta-analysis. ${ }^{23,24}$ Adjuvant! estimates have subsequently been validated using the British Columbia Breast Cancer Outcomes Unit database. ${ }^{25}$

The major advantage of Adjuvant is that it provides an in-the-office real-time tool to estimate recurrence risk and predict treatment benefit. The limitations of Adjuvant! are that it incorporates wide ranges of tumor size and number of lymph nodes, ER assessment is qualitative (either + or - ), and PR is not included, so a potentially more favorable $100 \%$ ER+ and 100\% PR-positive tumor is assessed the same as a $10 \% \mathrm{ER}+$ and PR-negative tumor. It also does not incorporate HER2 testing in computing prognosis and response to therapy.

\section{Multigene molecular assays}

Studies have demonstrated that multigene molecular panels can more accurately predict relapse than standard 
clinicopathologic criteria in both node-negative and nodepositive and hormone receptor-negative and -positive patients. ${ }^{26-28}$

\section{Oncotype DX ${ }^{\circledR}$}

Oncotype DX ${ }^{\circledR}$ (Genomic Health Inc, Redwood City, CA, USA) is the first assay to provide both prognostic estimates and predictions about response to therapy. Other available genomic assays had provided prognostic information, but not information to also guide treatment. ${ }^{8,29-31}$ This 21-gene assay (Oncotype DX Recurrence Score [RS]) uses high-throughput real-time, reverse-transcriptase polymerase chain reaction to quantify gene expression levels of 21 genes, including five control genes. The assay has been validated as a reliable quantifier of risk of distant recurrence in two independent data sets of women with node-negative ER+ breast cancer treated with tamoxifen. ${ }^{32,33}$ The RS has also been demonstrated to predict the benefit from adjuvant chemotherapy in this patient population in the NSABP B-20 trial and Southwest Oncology Group 8814 trial. ${ }^{34-38}$ The RS stratifies patients into low risk ( $\mathrm{RS}<18$ ), intermediate risk $(\geq 18$ but $<31$ ), and high risk ( $\mathrm{RS}$ score $\geq 31$ ) of distant recurrence. The disease-free survival at 10 years was $93.2 \%$ and $69.5 \%$ for the low-risk and high-risk groups, respectively. The RS provided predictive power independent of patient's age and tumor size. ${ }^{32}$

The Oncotype DX RS reveals the continuous biology of breast cancer. There are some underlying phenotypes that may correspond to low RS disease and high RS disease. ${ }^{37}$ There was a modest concordance with patient age. In a large ER+ patient cohort, an increased percentage of low RS has been observed in younger women, whereas older patients tend to have an increased percentage of high RS. ${ }^{39}$ The RS was also associated with PR ligand binding and poor grade, but with a significant degree of discordance. Data from the NSABP B-20 trial showed more T1ab tumors with low RS; however, in a validation cohort, approximately $16 \%$ of patients with $\mathrm{T} 1 \mathrm{ab}$ tumor had high $\mathrm{RS} .{ }^{40}$ There is a wide range of RS across patient's age, grade, node-negative, and node-positive status. ${ }^{39}$ This highlights the prognostic significance of 21 -gene array beyond traditional clinicopathologic phenotypes.

These RS distinctions help identify patients likely to derive benefit from systemic chemotherapy. The higher the score, the greater the risk of distant recurrence, with this subset obtaining a significant benefit from the addition of chemotherapy to tamoxifen. Conversely, the precision of the RS, with a tight confidence interval in the lower RS ranges, allows oncologists to confidently identify low-risk disease. Since a low RS value is associated with low risk of distant recurrence and lack of benefit from adding chemotherapy to tamoxifen, many oncologists can confidently recommend these patients forego chemotherapy. Among node-positive ER+ patients, Oncotype DX has also been found to significantly predict the benefit from adjuvant anthracycline-containing and nonanthracycline-containing adjuvant chemotherapy regimens. ${ }^{38}$ While many patients will benefit from chemotherapy, not all require anthracyclines, which have the added risk of cardiotoxicity and secondary malignancy. If some patients (ie, those with low RS) are not expected to get the added benefit of anthracyclines, they can be spared the added toxicity.

Approximately $25 \%$ of patients will fall into the intermediate RS range. Although the continuous nature of the assay allows oncologists to ascertain distant recurrence risk for these patients, there are multiple ways to apply the information. Patients with a "high" intermediate RS may be considered differently from those with a "low" intermediate RS value. Nonetheless, the information provided by the RS result can be helpful in making treatment decisions when viewed in the context of other patient-specific factors, including patient concern, age, tumor size and grade, and conditions that may increase the risk of chemotherapyassociated toxicity. ${ }^{32,33,37}$

\section{MammaPrint $^{\circledR}$}

MammaPrint $^{\circledR}$ (Agendia, Irvine, CA, USA) is a microarray gene expression based on differential gene expression of 70 genes that were significantly correlated with disease outcome in 78 frozen samples from lymph node-negative breast cancer $<5 \mathrm{~cm}$, patients $<55$ years at diagnosis, mostly without adjuvant systemic therapy. ${ }^{41}$ This RNA gene microarray provides a qualitative binary output (good or poor prognosis) using a relatively large number of genes compared to the Oncotype DX.

The 70-gene assay was validated in two studies with diverse patient populations, in terms of hormonal status, nodal status, and adjuvant therapy received. Both studies relied on convenience samples. ${ }^{42}$ In the first validation study, the patient population was relatively young and mixed in terms of nodal status (both node-negative and node-positive disease), ER status (both ER+ and ER-), and adjuvant treatment received. However, 61 of the 295 patients in the validation cohort were also used to develop the prognostic profile, which has the danger of overestimating the predictive potential of this assay. ${ }^{27}$ The second validation study included patients with characteristics different from those of patients in the first validation study. Although the patients in the 
second validation study were all node negative and untreated, the patients were mixed in terms of ER status. In addition, the primary end points differed between the two validation studies: the first measured risk of metastasis at 10 years and the second measured time to distant metastases. ${ }^{41,43}$

The benefit of chemotherapy was evaluated in a pooled analysis of clinical data from patients with a Mammaprint 70-gene assay who had received chemotherapy or endocrine therapy. The studies included in the analysis were retrospective and observational. Although there was a suggestion of benefit for chemotherapy added to endocrine therapy in high-risk patients, the level of evidence does not meet the threshold for determining clinical utility.

\section{Prosigna ${ }^{\circledR} / \mathrm{PAM} 50$}

Prosigna/PAM50 (NanoString Technologies Inc., Seattle, WA, USA) is a second-generation multigene assay, which utilizes 50 discriminator genes and eight controls to identify intrinsic breast cancer subtypes, that is, ER+/HER2 negative (luminal A/B), HER2 enriched, and basal like (ER-/HER2-), and tumors that have characteristics similar to normal breast tissue..$^{32,44-46}$ In retrospective analyses, these gene expression subtypes are associated with differing relapse-free survival and overall survival. Additionally, the PAM50 assay incorporates gene signatures associated with proliferation and pathologic tumor size (coded as $<2 \mathrm{~cm}$ or $>2 \mathrm{~cm}$ ). This generates a continuous "risk of relapse" score from 0 to 100 , with preassigned cutoffs for different risk groups based on the 10-year probability of recurrence: $<10 \%, 10 \%-20 \%$, and $>20 \%$ categorized as low, intermediate, and high risk, respectively.

Clinical validation studies for PAM50 included the Austrian Breast and Colorectal Cancer Study Group (ABCSG)-8 trial population of postmenopausal women with hormone receptor-positive breast cancer who had received 5 years of endocrine therapy alone in the form of 5 years of tamoxifen or 2 years of tamoxifen followed by 3 years of an aromatase inhibitor. Approximately $70 \%$ of the patients were $\mathrm{T} 1$ stage and node negative. The risk of relapse score accurately predicted the 10-year risk of distant metastasis.

In comparison with the Oncotype DX, using the TransATAC cohort, the PAM50 provided more prognostic information than the 21-gene-derived RS score. ${ }^{32,42,46}$ However, the PAM50 lacks high-level validation of the predictive benefit of chemotherapy.

\section{Mammostrat ${ }^{\circledR}$}

The Mammostrat ${ }^{\circledR}$ (Clarient Diagnostic Services, Inc, Aliso Viejo, CA, USA) test uses immunohistochemical assays measuring five genes in breast cancer cells, $S L C 7 A 5$, HTF9C, P53, NDRG1, and CEACAM5, to stratify patients on tamoxifen therapy into risk groups. The five markers are independent and do not directly measure either proliferation or hormone receptor status, so results may be interpreted in conjunction with conventional histopathologic features and hormone receptor status.

Clinical validation studies for Mammostrat included patients with both node-negative and node-positive disease. Ross et al used archival samples from tamoxifen-treated patients in NSABP B-14 and NSABP B-20 to validate the assay. ${ }^{47}$ Bartlett et al used archival samples collected from consecutive patients with $\mathrm{T} 1$ or T2, N0 or N1, and M0 disease at a single center. Only patients with ER+, node-negative or node-positive disease who had received hormonal therapy were included in the primary, prospectively defined analyses in this study. ${ }^{48}$ Although there was a significant association between patients stratified by Mammostrat and recurrencefree interval (RFI), distant RFI, and breast cancer-specific death, the low-risk and moderate-risk groups predict similar risk of recurrence for any of these outcome variables. In addition, the Mammostrat low-risk group had a relatively high RFI of $85 \%$.

Mammostrat is not clinically validated to predict chemotherapy benefit. Mammostrat identified high-risk and low-risk groups that both showed significant improvement in treatment with tamoxifen and chemotherapy. The moderaterisk group showed no significant benefit of chemotherapy and tamoxifen compared to tamoxifen alone. A test for an interaction between chemotherapy and risk group stratification was not significant $(P=0.13) .{ }^{47}$ In the "Tamoxifen versus Exemestane Adjuvant Multicenter" (TEAM) trial, the data showed no significant difference in distant relapse-free survival between the high- and low-risk stratified groups $(89 \%$ vs $82 \%){ }^{49}$ The 5 -year risk of disease recurrence or death was $11 \%$, which is higher than expected for a low-risk population. Based on these findings, the authors concluded that Mammostrat has no independent prognostic ability to predict relapse within 5 years. ${ }^{50}$

\section{Key differences between genomic assays}

A high rate of discordance has been observed between the MammaPrint and Oncotype DX assays. The results of a small, single-institution study conducted in France on patients who had both the MammaPrint assay and Oncotype assay showed that of patients in the MammaPrint high-risk group, ten of the $22(45 \%)$ had a low RS, indicating minimal 
benefit from chemotherapy and only one of the $22(5 \%)$ had a high RS. ${ }^{51}$ This discordance in risk assessment was reproduced in another study by Shivers et al, which directly compared MammaPrint, Oncotype DX, and Mammostrat. In this study, 33.3\% of MammaPrint high-risk patients were classified as low risk by Oncotype DX and conversely 5.6\% of low-risk MammaPrint patients were classified as high-risk RS. ${ }^{52}$ A direct comparison of PAM50 and Oncotype DX showed concordance for high RS and luminal B (high risk), as well as luminal A and low RS. However, more patients in the intermediate RS group were reclassified by PAM50 as low risk, suggesting that PAM50 may augment prognostic information provided by Onctoype DX alone. ${ }^{53}$

Although each of these assays can reliably predict risk of recurrence, only the Oncotype DX has been validated at this point to predict the benefit of chemotherapy. Clinicians should use caution and consider the limitations of these tests when considering which assay to use in decision-making about treatment.

\section{Future studies}

As previously discussed, the major limitation of the genomic assays is that although validated in large varied cohorts, all studies to date have been retrospective. The Microarray in Node negative Disease may Avoid ChemoTherapy (MINDACT) trial is the first major prospective validation of molecular assays. It is a large, multinational, and multicenter randomized controlled trial that started accrual in 2007 and completed recruitment in July 2011. In this study 6,000 patients with breast cancer having up to three positive lymph nodes will have risk assessed by both AdjuvantOnline! and the 70-gene profile, MammaPrint. If both assays classify the patient as low risk, then chemotherapy will be withheld; if both classify as high risk, then adjuvant chemotherapy will be given. If the results are discordant, the patient will be randomized to follow either clinocopathologic method or genomic results. A key group of women being assessed are those classified as high risk by clinicopathologic method and low risk by genomic assay and randomized to follow the low-risk genomic results. Secondary randomizations are incorporated into the trial design of MINDACT to evaluate nonanthracycline-based adjuvant chemotherapy regimens and first-line therapies for adjuvant endocrine therapy. ${ }^{54}$ The hypothesis is that MammaPrint will reduce the percentage of women receiving adjuvant chemotherapy based on traditional risk classifications by $10 \%-20 \%$ without any adverse effect on outcome. A planned pilot study of 800 patients confirmed that despite the logistical complexity of this trial, it is feasible. Among these patients, $48 \%$ were concordant low risk by both assays and $24.5 \%$ were concordant high risk, with a discordant rate of $27 \%, 17.6 \%$ of whom are clinically high but genomic low risk. These are the patients who could potentially be spared chemotherapy. ${ }^{55}$ The results of this study will define clinical utility of the "omics" world in clinical practice. The findings will help determine the predictive value of MammaPrint and the benefit of treating intermediate-risk patients with adjuvant chemotherapy.

The TAILORx and RxPONDER trials are being conducted in parallel to assess the benefit of adjuvant chemotherapy in addition to endocrine therapy in patients with ER+ breast cancer having intermediate prognosis by Oncotype RS. In the TAILORx trial, patients with node-negative, HRpositive breast cancer classified as intermediate risk are being randomized to receive chemotherapy or no chemotherapy. ${ }^{56}$ The RxPONDER trial will confirm SWOG 8814 trial data for women with ER+, 1-3 LN node-positive disease treated with chemotherapy or not based on risk scores. The findings will help determine the benefit of treating patients (node negative and node positive) at intermediate risk with adjuvant chemotherapy. ${ }^{57}$

\section{Conclusion}

In this review, we identify the traditional histopathologic features, clinicopathologic risk prediction models, and commercially available genomic assays used in clinical practice for patients with early breast cancer to assess prognosis and increase precision of adjuvant therapy. We limited our scope to commercially available assays that are most commonly used in the clinical setting in the USA and Europe - Oncotype DX, MammaPrint, PAM50, and Mammostrat. Although the development and validation of these assays are different, they all provide information on prognosis with the exception of the Oncotype DX, which also provides predictive information. A major drawback of these assays is that the validation studies are largely based on retrospective cohorts of patients treated according to the existing clinical guidelines at the time of diagnosis. The results of the large prospective trials - MINDACT, TAILORx, and RxPONDER - will provide rigorous data to guide therapy that will improve outcomes by more accurately identifying patients who can be spared chemotherapy and associated toxicity without compromising survival and conversely patients in whom adjuvant chemotherapy portends a survival advantage.

\section{Disclosure}

The authors report no conflicts of interest in this work. 


\section{References}

1. Bishop HM, Blamey RW. A suggested classification of breast pain. Postgrad Med J. 1979;55(Suppl 5):59-60.

2. Haybittle JL, Blamey RW, Elston CW, et al. A prognostic index in primary breast cancer. Br J Cancer. 1982;45(3):361-366.

3. Williams C, Brunskill S, Altman D, et al. Cost-effectiveness of using prognostic information to select women with breast cancer for adjuvant systemic therapy. Health Technol Assess. 2006;10(34):iii-iv, ix-xi, $1-204$.

4. Goldhirsch A, Wood WC, Coates AS, et al; Panel Members. Strategies for subtypes - dealing with the diversity of breast cancer: highlights of the St Gallen International Expert Consensus on the Primary Therapy of Early Breast Cancer 2011. Ann Oncol. 2011;22(8):1736-1747.

5. Goldhirsch A, Glick JH, Gelber RD, Senn HJ. Meeting highlights: International Consensus Panel on the Treatment of Primary Breast Cancer. J Natl Cancer Inst. 1998;90(21):1601-1608.

6. Eifel P, Axelson JA, Costa J, et al. National Institutes of Health Consensus Development Conference Statement: adjuvant therapy for breast cancer, November 1-3, 2000. J Natl Cancer Inst. 2001;93(13):979-989.

7. McGuire WL. Breast cancer prognostic factors: evaluation guidelines. J Natl Cancer Inst. 1991;83(3):154-155.

8. Hayes DF. Do we need prognostic factors in nodal-negative breast cancer? Arbiter. Eur J Cancer. 2000;36(3):302-306.

9. Fisher ER, Costantino J, Fisher B, Redmond C. Pathologic findings from the National Surgical Adjuvant Breast Project (Protocol 4). Discriminants for 15-year survival. National Surgical Adjuvant Breast and Bowel Project Investigators. Cancer. 1993;71(6 Suppl):2141-2150.

10. Fisher B, Redmond C, Poisson R, et al. Eight-year results of a randomized clinical trial comparing total mastectomy and lumpectomy with or without irradiation in the treatment of breast cancer. $N$ Engl J Med. 1989;320(13):822-828.

11. Fisher ER, Anderson S, Redmond C, Fisher B. Pathologic findings from the National Surgical Adjuvant Breast Project protocol B-06. 10-Year pathologic and clinical prognostic discriminants. Cancer. 1993; 71(8):2507-2514.

12. Rosen PP, Groshen S, Saigo PE, Kinne DW, Hellman S. Pathological prognostic factors in stage I (T1N0M0) and stage II (T1N1M0) breast carcinoma: a study of 644 patients with median follow-up of 18 years. J Clin Oncol. 1989;7(9):1239-1251.

13. Fisher B, Bryant J, Wolmark N, et al. Effect of preoperative chemotherapy on the outcome of women with operable breast cancer. J Clin Oncol. 1998;16(8):2672-2685.

14. Bear HD, Anderson S, Smith RE, et al. Sequential preoperative or postoperative docetaxel added to preoperative doxorubicin plus cyclophosphamide for operable breast cancer:National Surgical Adjuvant Breast and Bowel Project Protocol B-27. J Clin Oncol. 2006;24(13):2019-2027.

15. Rakha EA, Lee AH, Evans AJ, et al. Tubular carcinoma of the breast: further evidence to support its excellent prognosis. J Clin Oncol. 2010; 28(1):99-104.

16. Caldarella A, Buzzoni C, Crocetti E, et al. Invasive breast cancer: a significant correlation between histological types and molecular subgroups. J Cancer Res Clin Oncol. 2013;139(4):617-623.

17. Dieci MV, Orvieto E, Dominici M, Conte P, Guarneri V. Rare breast cancer subtypes: histological, molecular, and clinical peculiarities. Oncologist. 2014;19(8):805-813.

18. Berry DA, Cirrincione C, Henderson IC, et al. Estrogen-receptor status and outcomes of modern chemotherapy for patients with node-positive breast cancer. JAMA. 2006;295(14):1658-1667.

19. Voogd AC, Nielsen M, Peterse JL, et al; Danish Breast Cancer Cooperative Group; Breast Cancer Cooperative Group of the European Organization for Research and Treatment of Cancer. Differences in risk factors for local and distant recurrence after breast-conserving therapy or mastectomy for stage I and II breast cancer: pooled results of two large European randomized trials. J Clin Oncol. 2001;19(6):1688-1697.

20. Cooke T, Reeves J, Lanigan A, Stanton P. HER2 as a prognostic and predictive marker for breast cancer. Ann Oncol. 2001;12(Suppl 1): S23-S28.
21. Bonadonna G, Valagussa P, Moliterni A, Zambetti M, Brambilla C. Adjuvant cyclophosphamide, methotrexate, and fluorouracil in nodepositive breast cancer: the results of 20 years of follow-up. $N$ Engl J Med. 1995;332(14):901-906.

22. Ravdin PM, Siminoff LA, Davis GJ, et al. Computer program to assist in making decisions about adjuvant therapy for women with early breast cancer. J Clin Oncol. 2001;19(4):980-991.

23. Tamoxifen for early breast cancer: an overview of the randomised trials. Early Breast Cancer Trialists' Collaborative Group. Lancet. 1998;351(9114):1451-1467.

24. Polychemotherapy for early breast cancer: an overview of the randomised trials. Early Breast Cancer Trialists' Collaborative Group. Lancet. 1998;352(9132):930-942.

25. Olivotto IA, Bajdik CD, Ravdin PM, et al. Population-based validation of the prognostic model ADJUVANT! For early breast cancer. J Clin Oncol. 2005;23(12):2716-2725.

26. Chang HY, Nuyten DS, Sneddon JB, et al. Robustness, scalability, and integration of a wound-response gene expression signature in predicting breast cancer survival. Proc Natl Acad Sci U S A. 2005;102(10): 3738-3743.

27. van de Vijver MJ, He YD, van't Veer LJ, et al. A gene-expression signature as a predictor of survival in breast cancer. $N$ Engl J Med. 2002;347(25):1999-2009.

28. Foekens JA, Atkins D, Zhang Y, et al. Multicenter validation of a gene expression-based prognostic signature in lymph node-negative primary breast cancer. J Clin Oncol. 2006;24(11):1665-1671.

29. Bryant J, Fisher B, Gunduz N, Costantino JP, Emir B. S-phase fraction combined with other patient and tumor characteristics for the prognosis of node-negative, estrogen-receptor-positive breast cancer. Breast Cancer Res Treat. 1998;51(3):239-253.

30. Henderson IC, Patek AJ. The relationship between prognostic and predictive factors in the management of breast cancer. Breast Cancer Res Treat. 1998;52(1-3):261-288.

31. Esteva FJ, Hortobagyi GN. Prognostic molecular markers in early breast cancer. Breast Cancer Res. 2004;6(3):109-118.

32. Paik S, Shak S, Tang G, et al. A multigene assay to predict recurrence of tamoxifen-treated, node-negative breast cancer. $N$ Engl J Med. 2004;351(27):2817-2826.

33. Habel LA, Shak S, Jacobs MK, et al. A population-based study of tumor gene expression and risk of breast cancer death among lymph node-negative patients. Breast Cancer Res. 2006;8(3):R25.

34. Fisher B, Costantino J, Redmond C, et al. A randomized clinical trial evaluating tamoxifen in the treatment of patients with node-negative breast cancer who have estrogen-receptor-positive tumors. $N$ Engl J Med. 1989;320(8):479-484.

35. Fisher B, Dignam J, Wolmark N, et al. Tamoxifen and chemotherapy for lymph node-negative, estrogen receptor-positive breast cancer. J Natl Cancer Inst. 1997;89(22):1673-1682.

36. Fisher B, Jeong JH, Bryant J, et al; National Surgical Adjuvant Breast and Bowel Project Randomised Clinical Trials. Treatment of lymphnode-negative, oestrogen-receptor-positive breast cancer: long-term findings from National Surgical Adjuvant Breast and Bowel Project randomised clinical trials. Lancet. 2004;364(9437):858-868.

37. Paik S, Tang G, Shak S, et al. Gene expression and benefit of chemotherapy in women with node-negative, estrogen receptor-positive breast cancer. J Clin Oncol. 2006;24(23):3726-3734.

38. Albain KS, Barlow WE, Shak S, et al; Breast Cancer Intergroup of North America. Prognostic and predictive value of the 21-gene recurrence score assay in postmenopausal women with node-positive, oestrogenreceptor-positive breast cancer on chemotherapy: a retrospective analysis of a randomised trial. Lancet Oncol. 2010;11(1):55-65.

39. Lieberman N, Baehner FL, Soussan-Gutman L, et al. Evaluation of Recurrence Score, Nodal Status and Traditional Clinicopathologic Metrics in a Large ER Positive Patient Cohort. Providence, RI: European Society of Medical Oncology; 2011 [Abstract \#1420].

40. Ravdin P. Controversies in Early Breast Cancer. SABCS; San Antonio, CA, USA 2011. 
41. van't Veer LJ, Dai H, van de Vijver MJ, et al. Gene expression profiling predicts clinical outcome of breast cancer. Nature. 2002;415(6871): 530-536.

42. Simon RM, Paik S, Hayes DF. Use of archived specimens in evaluation of prognostic and predictive biomarkers. J Natl Cancer Inst. 2009; 101(21):1446-1452.

43. Buyse M, Loi S, van't Veer L, et al; TRANSBIG Consortium. Validation and clinical utility of a 70-gene prognostic signature for women with node-negative breast cancer. J Natl Cancer Inst. 2006; 98(17):1183-1192.

44. Nielsen TO, Parker JS, Leung S, et al. A comparison of PAM50 intrinsic subtyping with immunohistochemistry and clinical prognostic factors in tamoxifen-treated estrogen receptor-positive breast cancer. Clin Cancer Res. 2010;16(21):5222-5232.

45. Parker JS, Mullins M, Cheang MC, et al. Supervised risk predictor of breast cancer based on intrinsic subtypes. J Clin Oncol. 2009;27(8): 1160-1167.

46. Dowsett M, Sestak I, Lopez-Knowles E, et al. Comparison of PAM50 risk of recurrence score with oncotype DX and $\mathrm{IHC} 4$ for predicting risk of distant recurrence after endocrine therapy. J Clin Oncol. 2013; 31(22):2783-2790.

47. Ross DT, Kim CY, Tang G, et al. Chemosensitivity and stratification by a five monoclonal antibody immunohistochemistry test in the NSABP B14 and B20 trials. Clin Cancer Res. 2008;14(20):6602-6609.

48. Bartlett JM, Thomas J, Ross DT, et al. Mammostrat as a tool to stratify breast cancer patients at risk of recurrence during endocrine therapy. Breast Cancer Res. 2010;12(4):R47.

49. Bartlett JM, Bloom KJ, Piper T, et al. Mammostrat as an immunohistochemical multigene assay for prediction of early relapse risk in the tamoxifen versus exemestane adjuvant multicenter trial pathology study. $J$ Clin Oncol. 2012;30(36):4477-4484.

50. Bartlett JMS, Bloom KJ, Goldstein NS, et al. Mammostrat ${ }^{\circledR}$ as an Immunohistochemical Multigene Assay for Prediction of Early Relapse Risk in Postmenopausal Early Breast Cancer: Preliminary Data of the TEAM Pathology Study. SABCS 2010. Poster/Abstract: P3-10-33.
51. Clough KB, Poulet B, Jamshidian F, et al. Risk Classification of Early Stage Breast Cancer as Assessed by MammaPrint and Oncotype DX Genomic Assay. SABCS 2012. Poster/Abstract: p6-07-03.

52. Shivers SC, Clark L, Esposito N, H, et al. Direct comparison of risk classification between MammaPrint $^{\mathbb{R}}$, Oncotype DX ${ }^{\circledR}$ and MammoStrat ${ }^{\mathbb{R}}$ assays in patients with early stage breast cancer. SABCS 2013. Poster/ Abstract: P6-06-02.

53. Kelly CM, Bernard PS, Krishnamurthy S, et al. Agreement in risk prediction between the 21-gene recurrence score assay (Oncotype DX(R)) and the PAM50 breast cancer intrinsic Classifier in early-stage estrogen receptor-positive breast cancer. Oncologist. 2012;17(4):492-498.

54. Cardoso F, Piccart-Gebhart M, Van't Veer L, Rutgers E, Consortium T. The MINDACT trial: the first prospective clinical validation of a genomic tool. Mol Oncol. 2007;1(3):246-251.

55. Rutgers E, Piccart-Gebhart MJ, Bogaerts J, et al. The EORTC 10041/ BIG 03-04 MINDACT trial is feasible: results of the pilot phase. Eur J Cancer. 2011;47(18):2742-2749.

56. Hormone therapy with or without combination chemotherapy in treating women who have undergone surgery for node negative breast cancer (the TAILORx Trial). Clinical Trial ID: NCT00310180. Available from: https:/clinicaltrials.gov/ct2/show/NCT00310180. Accessed June 24, 2015.

57. A phase III, radomized clinical trial of standard adjuvant endocrine therapy $+/-$ chemotherapy in patients with $1-3$ positive nodes, hormone receptor-positive and HER2- negative breast cancer with a recurrence score (RS) of 25 or less. RxPONDER: a clinical trial RX for positive node, endocrine responsive breast cancer. Clinical Trial ID NCT01272037. Available from: https://clinicaltrials.gov/ct2/show/ NCT01272037.
Breast Cancer: Targets and Therapy

\section{Publish your work in this journal}

Breast Cancer: Targets and Therapy is an international, peerreviewed open access journal focusing on breast cancer research, identification of therapeutic targets and the optimal use of preventative and integrated treatment interventions to achieve improved outcomes, enhanced survival and quality of life for the cancer patient.

\section{Dovepress}

View the full aims and scopes of this journal here. The manuscript management system is completely online and includes a very quick and fair peer-review system, which is all easy to use. Visit http:// www.dovepress.com/testimonials.php to read real quotes from published authors. 